\section{Cyclische Dimethylzinn-diamide durch Umaminierung}

O. J. Scherer, J. Schmidt, J. Wokulat und M. Schmidt

Institut für Anorganische Chemie der Universität Marburg

(Z. Naturforschg. 20 b, 183-184 [1965] ; eingeg. am 7. November 1964)

Im Zusammenhang mit dem Studium von Zinn-Stickstoffbindungen ${ }^{1}$ haben wir gefunden, daß Dimethylzinnbis-diäthylamid $^{2}$ (I) (ähnlich wie z. B. entsprechende Borverbindungen ${ }^{3}$ ) mit bifunktionellen sekundären Aminen leicht zu cyclischen Zinn-diamiden reagiert:
So entsteht aus I und $N, N^{\prime}$-Dimethyl-trimethylendiamin in 75-proz. Ausbeute 2.2'-Dimethyl-1.3-dimethyl-1.3diaza-2-stannacyclohexan (II) $\left(R=\mathrm{CH}_{3}, n=1\right)$ als wasserklare Flüssigkeit vom Schmp. $1-3^{\circ}$. Das entsprechende Cyclopentanderivat $(n=0)$ konnten wir nicht erhalten.

Besonders leicht verlaufen die Umaminierungen mit $N, N^{\prime}$-Bis-trimethylsilyldiaminen ${ }^{4,5}$, die zu $2.2^{\prime}$-Dimethyl1.3-bis-trimethylsilyl-1.3-diaza-2-stannacyclopentan (III) $\left(\mathrm{R}=\left(\mathrm{CH}_{3}\right)_{3} \mathrm{Si}, n=0\right)$ bzw. -cyclohexan (IV), $\quad(\mathrm{R}=$ $\left.\left(\mathrm{CH}_{3}\right)_{3} \mathrm{Si}, n=1\right)$ bzw. -cycloheptan (V) $\left(\mathrm{R}=\left(\mathrm{CH}_{3}\right)_{3} \mathrm{Si}\right.$, $n=2$ ) führen. (Die III entsprechende Si-Verbindung wurde bereits beschrieben ${ }^{6}$ ).

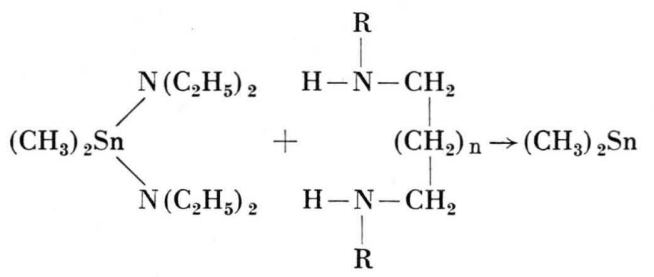

I<smiles>[R]N(C)CCCCCCN</smiles>

II - V

$\left(\mathrm{R}=\mathrm{CH}_{3},\left(\mathrm{CH}_{3}\right)_{3} \mathrm{Si} ; n=0,1,2\right)$.

Auch $N . N^{\prime}$-Bis-trimethylsilyl-o-phenylendiamin ${ }^{5}$ reagiert in gleicher Weise und liefert mit I nach<smiles>CCNCCCCNc1ccccc1N[Si]C(C)(C)C</smiles>

in guten Ausbeuten das erwartete Produkt VI in Form grünlicher Kristalle, die sich selbst unter Stickstoff oberflächlich langsam schwarz färben (bei Zutritt geringster Luftmengen sofort!).

Alle beschriebenen Verbindungen sind erwartungsgemäß hydrolyseempfindlich. Ihre ProtonenresonanzSpektren entsprechen gut den Erwartungen. Sie eignen sich ausgezeichnet zur Charakterisierung.

\section{Experimentelles}

Die Umsetzungen werden ohne Lösungsmittel in trockener Stickstoffatmosphäre in einer einfachen Destillationsapparatur durchgeführt (Ölbadheizung, Magnetrührung). Im folgenden werden die jeweils eingesetzten Mengen Ausgangsmaterial, die Reaktionstemperatur, die Reaktionszeit und die Ausbeuten angegeben.

\begin{tabular}{|c|c|c|c|c|c|c|c|c|}
\hline \multicolumn{5}{|c|}{$\begin{array}{c}\text { Kopplungskonstanten } J \\
{[\mathrm{~Hz}]}\end{array}$} & \multicolumn{4}{|c|}{$\begin{array}{l}\text { Chemische Verschiebung } \\
{[\mathrm{cps}]}\end{array}$} \\
\hline No. & $\mathrm{JSn}^{117}-\mathrm{CH}_{3}$ & $\mathrm{JSn}^{119}-\mathrm{CH}_{3}$ & $\mathrm{JSn}^{117}-\mathrm{CH}_{2-}$ & $\mathrm{JSn}^{119}-\mathrm{CH}_{2}-$ & $\delta \mathrm{SiCH}_{3}$ & $\delta \mathrm{SnCH}_{3}$ & $\delta \mathrm{NCH}_{3}$ & $\delta \mathrm{CH}_{2}$ \\
\hline $\begin{array}{r}\text { II } \\
\text { III } \\
\text { IV } \\
\text { V } \\
\text { VI }\end{array}$ & $\begin{array}{l}55,5 \\
58,0 \\
58,0 \\
58,0 \\
59,5\end{array}$ & $\begin{array}{l}58,5 \\
60,5 \\
61,0 \\
60,5 \\
62,0\end{array}$ & 43,0 & 45,5 & $\begin{array}{l} \pm 0 \\
\pm \quad 0 \\
\pm \quad 0 \\
-11,5\end{array}$ & $\begin{array}{l}-20,5 \\
-23,0 \\
-21,5 \\
-21,0 \\
-39,5\end{array}$ & $-155,0$ & $-174,0$ \\
\hline
\end{tabular}

Tab. 1. ${ }^{1} \mathrm{H}$-NMR-Spektren (5-proz. Lösung in $\mathrm{CCl}_{4}$, Varian A 60; TMS als int. Standard).

1 O. J. Scherer, J. F. Schmidt u. M. Schmidt, Z. Naturforschg. 19 b, 447 [1964].

2 E. Wiberg u. R. Rieger, G. P. 1, 121050 (1962), Dissertation R. Rieger, München 1962.

3 Zum Beispiel K. Niedenzu, P. Fritz u. J. W. Dawson, Inorg. Chem. 3, 1077 [1964].
${ }^{4}$ F. A. Henglein u. K. Lienhard, Makromolekulare Chem. 32, 218 [1959].

5 L. Birkofer, H. P. Kühltau u. A. Ritter, Chem. Ber. 93, 2810 [1960].

${ }^{6}$ D. Kummer u. E. G. Rochow, Z. anorg. allg. Chem. 321, 21 [1963]. 
Verbindung II: $3,7 \mathrm{~g}$ (13 mMol) I+ $1,3 \mathrm{~g}(13 \mathrm{mMol})$ $N, N^{\prime}$-Dimethyl-trimethylendiamin. Zwischen $70-100^{\circ}$ destilliert innerhalb einer Stde. das gebildete Diäthylamin ab. Der Rückstand wird fraktioniert destilliert. Ausbeute $2,4 \mathrm{~g}$ ( $77 \%$ d. Th.), Sdp. ${ }_{18} 88-90^{\circ}$, Schmp. $1-3^{\circ}$.

$\mathrm{C}_{7} \mathrm{H}_{18} \mathrm{~N}_{2} \mathrm{Sn}(248,7) \quad$ Ber. C $33,7 \quad \mathrm{H} \quad 7,2 \quad \mathrm{~N} 11,2$, Gef. C 33,4 H 7,4 N 11,0.

Verbindung III: $2,5 \mathrm{~g}(8,5 \mathrm{mMol}) \mathrm{I}+1,73 \mathrm{~g}$ $(8,5 \mathrm{mMol}) \quad N, N^{\prime}$-Bis-trimethyl-silyl-äthylendiamin. 120 bis $130{ }^{\circ} \mathrm{C}(1 / 2$ Stde.), Ausbeute $2,3 \mathrm{~g}$ (77\% d. Th.). Sdp. $18121-123^{\circ} \mathrm{C}$, Schmp. $14-16^{\circ} \mathrm{C}$. $\mathrm{C}_{10} \mathrm{H}_{28} \mathrm{~N}_{2} \mathrm{Si} 2 \mathrm{Sn}(350,7)$

Ber. C 34,2 H 8,0 N 8,0,

Gef. C 34,5 H 8,1 N 7,8 (MG. 331,0).

Verbindung $I V: 3,7 \mathrm{~g}(13 \mathrm{mMol}) \mathrm{I}+2,75 \mathrm{~g}(13 \mathrm{mMol})$ $N, N^{\prime}$ Bis-trimethylsilyl-trimethylendiamin. $110-125{ }^{\circ} \mathrm{C}$ (1 Stde.), Ausbeute 3,0 g (65\% d. Th.). Sdp.16 136 bis $138{ }^{\circ} \mathrm{C}$; Schmp. $-2{ }^{\circ} \mathrm{C}$.
$\mathrm{C}_{11} \mathrm{H}_{30} \mathrm{~N}_{2} \mathrm{Si}_{2} \mathrm{Sn}(364,7)$

Ber. C 36,2 H 8,2 N 7,7,

Gef. C 36,5 H $8,1 \quad$ N 7,5.

Verbindung $V: 3,7 \mathrm{~g}(13 \mathrm{mMol}) \mathrm{I}+2,9 \mathrm{~g}(13 \mathrm{mMol})$ $N, N^{\prime}$ Bis-trimethylsilyl-tetramethylendiamin. 110 bis $130{ }^{\circ} \mathrm{C}$ (1 Stde.), Ausbeute $3,0 \mathrm{~g}(63 \%$ d. Th.). Sdp. 0,1 $70-71{ }^{\circ} \mathrm{C}$.

$\mathrm{C}_{12} \mathrm{H}_{32} \mathrm{~N}_{2} \mathrm{Si}_{2} \mathrm{Sn}(378,7)$

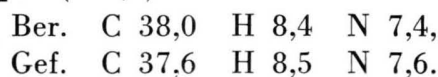

Verbindung VI: $3,7 \mathrm{~g}(13 \mathrm{mMol}) \mathrm{I}+3,2 \mathrm{~g}(13 \mathrm{mMol})$ $N, N^{\prime}$-Bis-trimethylsilyl-o-phenylendiamin. $\quad 80-110^{\circ} \mathrm{C}$ (1 Stde.; Schwarzfärbung), Ausbeute 3,5 g (70\% d. Th.). Sdp. $0,1114-117^{\circ} \mathrm{C}$; Schmp. $\sim 75^{\circ} \mathrm{C}$.

$\mathrm{C}_{14} \mathrm{H}_{28} \mathrm{~N}_{2} \mathrm{Si}_{2} \mathrm{Sn}(398,7)$

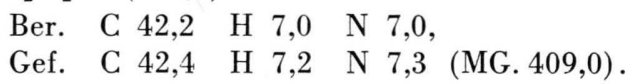

\section{Über Aromatenkomplexe von Metallen LXXXIII ${ }^{1}$}

\section{Bis-(cyclopentadienyl-eisen-dicarbonyl)-jod- Kation und seine Reaktion mit Elektronen- donatoren}

Ernst Otto Fischer und Erwin Moser

Anorganisch-chemisches Laboratorium der Technischen Hochschule München und Institut für Anorganische Chemie der Universität München

(Z. Naturforschg. 20 b, 184-185 [1965] ; eingeg. am 23. November 1964)

Vor kurzem konnten wir über das Hexafluorophosphat eines zweikernigen Bis-(cyclopentadienyl-eisen-dicarbonyl) -brom-Kations berichten, das bei der Reaktion von $\mathrm{C}_{5} \mathrm{H}_{5} \mathrm{Fe}(\mathrm{CO}){ }_{2} \mathrm{Br}$ mit $\mathrm{AlBr}_{3}$ in fl. $\mathrm{SO}_{2}$ entsteht ${ }^{2}$. Es gelang uns inzwischen unter verschärften Bedingungen, durch Umsetzung von $\mathrm{C}_{5} \mathrm{H}_{5} \mathrm{Fe}(\mathrm{CO})_{2} \mathrm{~J}$ mit $\mathrm{AlCl}_{3}$ in der Schmelze bei $60^{\circ}$ auch das entsprechende Jod-Kation zu erhalten. Dieses wurde wieder als Hexafluorophosphat aus wäßriger Lösung gefällt. Bereits bei anderen Reaktionen war vom Chlor zum Jod eine Abnahme der Tendenz, als Anion auszutreten, beobachtet worden ${ }^{3}$. Auch die geringe Neigung zur Bildung von Jodoaluminaten ${ }^{4}$ dürfte ein Grund für die benötigten energischeren Reaktionsbedingungen bei der Darstellung sein. Beim Umfällen aus Aceton mit Äther erhält man das Bis - (cyclopentadienyl-eisen-dicarbonyl) - jod-hexafluorophosphat $\left[\mathrm{C}_{5} \mathrm{H}_{5} \mathrm{Fe}(\mathrm{CO}){ }_{2}-\mathrm{J}-(\mathrm{CO})_{2} \mathrm{FeC}_{5} \mathrm{H}_{5}\right] \mathrm{PF}_{6}$ in dunkelroten Kristallen.

1 LXXXII Mitteil.: E. O. Fischer u. G. Huttner, J. organometal. Chem. (im Erscheinen).

2 E. O. Fischer u. E. Moser, J. organometal. Chem. (im Erscheinen).

${ }^{3}$ K. K. Joshi, P. L. Pauson u. W. H. Stubbs, J. organometal. Chem. 1, 51 [1963].

$$
\begin{aligned}
& \mathrm{C}_{14} \mathrm{H}_{10} \mathrm{~F}_{6} \mathrm{Fe}_{2} \mathrm{JO}_{4} \mathrm{P}(625,8) \\
& \text { Ber. C } 26,87 \text { H } 1,16 \text { F } 18,22 \text { Fe } 17,85 \\
& \text { J } 20,28 \text { P } 4,95 \text {, } \\
& \text { Gef. C 26,92 H 1,54 F 18,90 Fe 17,51 } \\
& \text { J 18,60 P 5,20. }
\end{aligned}
$$

Der diamagnetische Komplex ist bis $111^{\circ}$ an der Luft stabil (die Bromverbindung bis $80^{\circ}$ ), zersetzt sich jedoch in Lösung. Daher zeigt das ${ }^{1} \mathrm{H}$-NMR-Spektrum in Aceton (6-proz.) drei Signale bei 314, 334 und $338^{5}$ im Verhältnis $9: 122: 7$; nach 24 Stdn. beträgt das Verhältnis nur mehr $9: 33: 7$. Das Signal bei 314 kann wohl dem $\mathrm{C}_{5} \mathrm{H}_{5} \mathrm{Fe}(\mathrm{CO})_{2} \mathrm{~J}$, das in Aceton (5-proz.) bei 313 absorbiert, zugeschrieben werden. Das IR-Spektrum ${ }^{6}$ zeigt die Normalschwingungen des $\pi$-gebundenen, symmetrischen $\mathrm{C}_{5} \mathrm{H}_{5}$-Ringes bei $3125(\mathrm{~s}), 2915(\mathrm{w})$, $1425(\mathrm{~s}), 1114(\mathrm{w}), 1007(\mathrm{~m}), 887(\mathrm{sh})$ und $845(\mathrm{~s}) \mathrm{cm}^{-1}$. Die Banden bei 864(sh) und 826(s) können dem $\mathrm{PF}_{6}{ }^{\ominus}$. Anion zugeordnet werden. Die CO-Valenzfrequenzen, die gegenüber dem $\mathrm{C}_{5} \mathrm{H}_{5} \mathrm{Fe}(\mathrm{CO})_{2} \mathrm{~J}$ kaum nach oben verschoben sind (2041 und $1984 \mathrm{~cm}^{-1}$ ), gibt Tab. 1 wieder. Außerdem treten Absorptionen bei $1359(\mathrm{w})$, 1070 (wsh), 1065 (w), 1016 (wsh), 959(w), 778 (wsh), $739(\mathrm{w}), 599(\mathrm{~s}) 560(\mathrm{~s}), 538(\mathrm{~s}), 476(\mathrm{~s}), 435(\mathrm{~m})$ und $366(\mathrm{~m}) \mathrm{cm}^{-1}$ auf. Das Spektrum entspricht damit bis auf das Fehlen der schwachen Schultern bei 907 und $893 \mathrm{~cm}^{-1}$ und der Banden bei 820 und $528 \mathrm{~cm}^{-1} \mathrm{dem}$ der analogen Bromverbindung

$$
\left[\mathrm{C}_{5} \mathrm{H}_{5} \mathrm{Fe}(\mathrm{CO})_{2}-\mathrm{Br}-(\mathrm{CO})_{2} \mathrm{FeC}_{5} \mathrm{H}_{5}\right] \mathrm{PF}_{6} .
$$

Aus der Ubereinstimmung der Spektren und den unten beschriebenen Reaktionen folgern wir, wie schon bei

4 S. Ahrland, Acta chem. scand. 10, 723 [1956].

5 Die Signale sind in Hertz gegen Tetramethylsilan als internen Standard nach niedrigeren Feldern gemessen.

${ }^{6}$ Die IR-Spektren sind, wenn es nicht anders vermerkt ist, in Nujol/Hostaflon aufgenommen. 